\title{
Can Metal-Organic Frameworks Be Used for Cannabis Breathalyzers?
}

\author{
Daniele Ongari, ${ }^{\dagger, \S \odot}$ Yifei Michelle Liu, ${ }^{\dagger, \ddagger \S}$ and Berend Smit ${ }^{*}, \dagger, \pitchfork \odot$
}

${ }^{\dagger}$ Laboratory of Molecular Simulation (LSMO), Institut des Sciences et Ingénierie Chimiques, École Polytechnique Fédérale de Lausanne (EPFL), Rue de l'Industrie 17, Sion, Valais CH-1951, Switzerland

${ }^{\ddagger}$ Department of Chemical and Biomolecular Engineering, University of California, Berkeley, California 94720, United States

Supporting Information

ABSTRACT: $\Delta^{9}$-Tetrahydrocannabinol (THC) is the principal psychoactive component of cannabis, and there is an urgent need to build lowcost and portable devices that can detect its presence from breath. Similarly to alcohol detectors, these tools can be used by law enforcement to determine driver intoxication and enforce safer and more regulated use of cannabis. In this work, we propose to use a class of microporous crystals, metal-organic frameworks (MOFs), to selectively adsorb THC that can be later detected using optical, electrochemical, or fluorescence-based sensing methods. We computationally screened more than 5000 MOFs, highlighting the materials that have the largest affinity with THC, as well as the highest selectivity against water, showing that it is thermodynamically feasible for MOFs to adsorb THC from humid breath. We propose and compare different models for THC and different

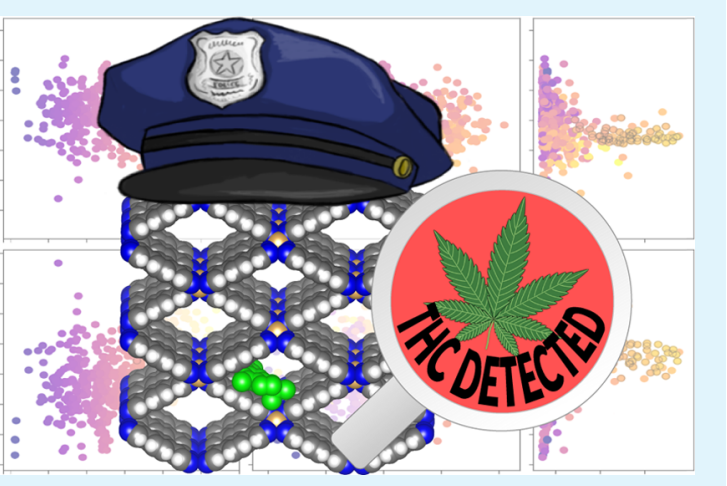
computational protocols to rank the promising materials, also presenting a novel approach to assess the permeability of a porous framework to nonspherical molecules. We identified three adsorption motifs in MOFs with high affinity to THC, which we refer to as "narrow channels", "thick walls", and "parking spots". Therefore, we expect our protocols and our findings to be generalizable for different classes of microporous materials and also for investigating the adsorption properties of other large molecules that, like THC, have a nonspherical shape.

KEYWORDS: tetrahydrocannabinol, THC, cannabis breathalyzer, metal-organic frameworks, gas adsorption

\section{INTRODUCTION}

Cannabis has in recent years seen increasing decriminalization for both recreational and medical use under some local and state jurisdictions. Since 2012, 10 U.S. states have legalized recreational use of cannabis and 33 states the medical use of the drug, coinciding with a shift in societal attitudes with regard to cannabis use. ${ }^{1,2}$ Immoderate cannabis use has been shown to have public health and safety implications, for example, an observable increase in risk of injury or fatality while driving under intoxication. ${ }^{1,3}$ As a result, increasing use of cannabis requires improved methods, similar to alcohol, for portable detection of cannabis intoxication in order to promote more consistent cannabis regulation and safer cannabis use.

The pharmacological effects of cannabis result from a variety of chemical compounds, including several hundred types of cannabinoid that can be found in the plant. The principal psychoactive component of cannabis is $\Delta^{9}$-tetrahydrocannabinol (THC), which affects motor and cognitive function and has been linked to increased risk of motor vehicle crashes. ${ }^{3-5}$ For roadside testing applications, breath-based testing of THC has the greatest potential as it is less invasive than testing of urine or blood.

Breath detection has other advantages as well. The window of detection of THC in breath (from 0.5 to up to $2 \mathrm{~h}$ after cannabis use) coincides with the period of peak impairment. ${ }^{6,7}$ THC and its metabolites can also be detected in blood and urine, but as cannabinoid compounds persist for several times longer in these bodily fluids they are not a reliable indicator of intoxication. ${ }^{2,8}$ Although the full window of driving impairment may extend longer than $2 \mathrm{~h},{ }^{5}$ the short detection window for the breath can be extended using methods that have increased sensitivity. ${ }^{9}$

THC sensing from breath is challenging because it has a very low vapor pressure $\left(1.15 \times 10^{-4} \mathrm{~Pa}\right.$ at $40{ }^{\circ} \mathrm{C}$, i.e., $10^{8}$ times lower than the vapor pressure of ethanol at the same temperature, $17.877 \mathrm{kPa}) .{ }^{10-12}$ Successful breath assays of THC to date have not relied on vapor-phase THC but rather have required that picogram amounts of THC be collected from aerosol droplets in the breath that are expelled when the subject breathes out. ${ }^{7,13}$ Other complicating factors of THC detection include its fast distribution from in the body from blood to tissue and the other chemically similar compounds that are present in cannabis, such as cannabinodiol and 11-nor9-carboxy-THC (THC-COOH), a metabolite of THC. Proven

Received: July 28, 2019

Accepted: August 27, 2019

Published: August 27, 2019 
methods to detect THC from breath have largely relied on mass spectrometry; ${ }^{5,13}$ more recently, a patent describing an early-stage fluorescence technology to detect THC was also published. ${ }^{14}$

In this work, we propose using metal-organic frameworks (MOFs) to adsorb THC prior to detection, which can be carried out using existing optical, ${ }^{15-17}$ electrochemical, ${ }^{18}$ or fluorescence-based $^{14}$ sensing methods. MOFs are porous, crystalline materials composed of metal nodes linked by organic ligands in a three-dimensional network. ${ }^{19-22}$ They have a high surface area for adsorption and a high degree of chemical tunability that makes possible the synthesis of a framework with ideal properties for a given application. The huge design space of MOFs is reflected in the large and growing number of experimentally synthesized MOFs that have been reported to date, nearing 100000 structures. ${ }^{23}$ With this chemical diversity of structures, MOFs have exhibited unmatched performance in applications such as adsorption, catalysis, and sensing. ${ }^{19,20}$

MOFs have been used as a detection medium for biologically relevant molecules such as chemical warfare agents and toxic industrial chemicals. ${ }^{24,25}$ Some applications for human breath analysis have been proposed, ${ }^{26,27}$ and there is an ongoing effort to understand if these materials can be combined for the detection of multiple components in the breath mixture. ${ }^{28}$ However, these studies mainly target small molecules (e.g., gas molecules and volatile organic compounds) and more investigation is needed to unravel the thermodynamics and kinetics of adsorption for biological molecules as big as THC. Moreover, sensing in biological systems poses particular challenges for MOFs, including interference of water with the adsorbate of interest and the thermal and chemical stability of MOFs in the presence of bodily fluids such as blood or saliva. One solution is to use hydrophobic materials that would minimize adsorption of water in order to detect the analyte or to adsorb water from the sample before it comes in contact with the MOF.

More generally, detection of larger biologically relevant molecules in MOFs is interesting because the adsorption energy of larger molecules may be more dependent on pore shape than adsorption of small gas molecules such as $\mathrm{N}_{2}$ and $\mathrm{CO}_{2}$. This allows for potentially higher selectivity when the pore shape can be optimized for an adsorbate molecule. Simulation of larger molecules can also pose computational challenges. Properties such as diffusion and adsorption become more expensive to compute, and the difference in computational time can be significant, especially when screening a library of structures for a particular application. Strategies to reduce computational cost while still capturing the decisive properties for an application can be useful. Toward this end, we tested two models of THC of different complexity.

With these adsorbate models, we investigated $645 \mathrm{MOF}$ structures that were filtered from a larger set of 5171 materials comprising the computation-ready experimental (CoRE) MOF database $^{29}$ and the MOF-74 analog database. ${ }^{30}$ Geometric analysis of the porous frameworks was carried out with the existing Zeo++ package $^{31}$ and a novel method using 2D projections of the adsorbate and pores to analyze pore accessibility toward asymmetric probe molecules. We used NVT and Widom insertion simulations to compute the adsorption energy and Henry coefficient of THC in these structures, and to determine whether using a simplified model (representing only the rigid aromatic head) would be an appropriate screening strategy for THC. We identified motifs of adsorption in the most promising structures. Finally, we computed the $\mathrm{THC} / \mathrm{H}_{2} \mathrm{O}$ selectivity in these materials and determined which might be suitable for THC detection in the presence of water.

\section{COMPUTATIONAL METHODS}

The atomic positions of the THC molecule were optimized with the CP2K package $^{32}$ using the PBE functional ${ }^{33}$ with Goedecker-TeterHutter (GTH) pseudopotentials, ${ }^{34}$ the DZVP-MOLOPT-GTH Gaussian basis set, ${ }^{35}$ and an auxiliary plane wave basis set with cutoff at 700 Ry. A $30 \times 30 \times 30 \AA \AA$ box was adopted with a wavelet-based Poisson solver to exclude periodic boundary conditions. The energy density from the converged calculation was used to compute DDEC06 point charges, using the software Chargemol. ${ }^{36}$

The model we used for THC is shown in Figure 1. We divided the THC structure in two sections: an $n$-pentyl group, which we refer to

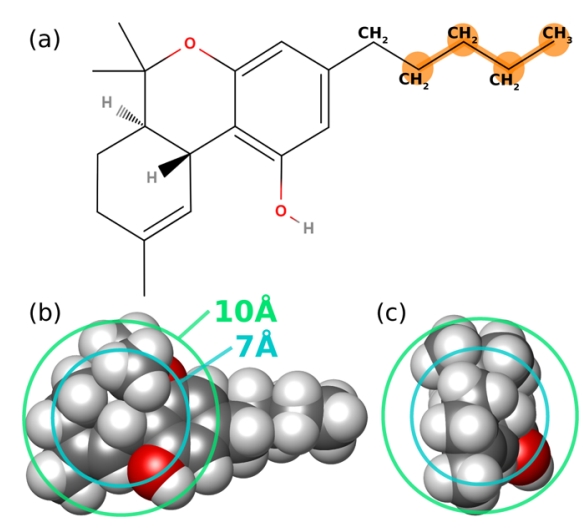

Figure 1. (a) Chemical sketch of the THC molecule: the "tail" section is highlighted in orange. It is composed by three $\mathrm{CH}_{2}$ and a terminal $\mathrm{CH}_{3}$ bead. The connecting $\mathrm{CH}_{2}$ bead and all other atoms are part of the "THC-head" model. All atoms that are not mentioned in the top figure as " $\mathrm{CH}_{2}$ " or " $\mathrm{CH}_{3}$ " beads are explicitly considered, as parametrized from UFF. ${ }^{37}$ Cross-sectional diameters of the DFToptimized THC molecule are shown (b) from a frontal and (c) lateral view. The diameters of the atoms are displayed as the Lennard-Jones $\sigma$ value from UFF: $2.57 \AA$ for $\mathrm{H}, 3.43 \AA$ for $\mathrm{C}$, and $3.12 \AA$ for $\mathrm{O}$.

hereafter as the "tail", and the remaining aromatic terpenoid group, which we refer to as the "head". Note that the $\alpha$-carbon of the 6carbon alkyl chain of THC is considered to be part of the head group, as the planar structure of the aromatic ring constrains the position of the $\alpha$-carbon with respect to the aromatic group. We employed a united-atom description of the flexible tail, treating it as a chain of four $\mathrm{CH}_{2}$ sites and a final $\mathrm{CH}_{3}$ site. For the head group, we used an all-atom description. The head group was kept rigid due to its constrained geometry.

We utilized a Lennard-Jones 12-6 potential with Lorentz-Berthelot mixing rules to model the dispersion interactions between THC and the framework. For the framework atoms and the all-atom head group, the Lennard-Jones $\varepsilon$ and $\sigma$ parameters were taken from the universal force field (UFF). ${ }^{37}$ For the coarse-grained tail group, the TraPPE force field parameters were used. ${ }^{38}$ Intramolecular interactions of the tail (bond, angle, and dihedral interactions) were also taken from the TraPPE force field. The carbon-carbon bonds of the hydrocarbon tail were kept rigid, with a bond length of $1.54 \AA$. The angles were described with a harmonic potential with an equilibrium angle of $114^{\circ}$ and a spring constant of $62500 \mathrm{~K} \mathrm{rad}^{-2}$, and the dihedrals with the TraPPE dihedral form

$$
\begin{aligned}
U= & p_{0}+p_{1}\left[1+\cos \phi_{i j k l}\right]+p_{2}\left[1-\cos 2 \phi_{i j k l}\right] \\
& +p_{3}\left[1+\cos 3 \phi_{i j k l}\right]
\end{aligned}
$$


where $p_{0} / k_{\mathrm{B}}, p_{1} / k_{\mathrm{B}}, p_{2} / k_{\mathrm{B}}$, and $p_{3} / k_{\mathrm{B}}$ were $0,335.03,-68.19$, and $791.32 \mathrm{~K}$, respectively. These parameters are consistent with the TraPPE united-atom model of $n$-alkanes. ${ }^{38}$

Short-range dispersion interactions had a cutoff distance of $12 \AA$ without applying tail corrections. The unit cells of all frameworks were replicated so that perpendicular widths between opposite faces were larger than twice the cutoff distance. The positions of the framework atoms were kept fixed for all simulations.

In this study, two models of THC are used: the THC-full model and the THC-head model. The THC-head model includes only the bulky head group of the THC molecule and is fully rigid. The THCfull model treats the aromatic head of the THC molecule as rigid, but also includes the hydrocarbon tail, which is flexible.

We used Monte Carlo (MC) methods to screen adsorption of THC in our materials library using both THC-head and THC-full models. The tail of the THC-full model was simulated using configurational-bias MC (CBMC). ${ }^{39}$ We ran Widom insertion simulations in the desolvated framework and NVT simulations with a loading of 1 molecule per simulation cell. In NVT simulations, we chose moves with equal probability of translation, rotation, reinsertion, and reinsertion-in-place, where the last is a CBMC move that applies to the THC-full model. For THC, we used blocking spheres generated in Zeo++ with a probe radius of $2.5 \AA$. This is smaller than the size of the THC molecule and was chosen conservatively in order not to exclude pores which might be accessible to THC considering a minimal vibration of the crystal. A more detailed method to determine pore accessibility is detailed in the following section. MC simulations were run in the RASPA package, ${ }^{40}$ considering a temperature of $309 \mathrm{~K}$, which is the human body temperature.

Our models and simulations were first tested in depth on a single framework, the well-studied Mg-MOF-74. ${ }^{4-44}$ This preliminary analysis led to the conclusions that Coulombic interactions can be neglected for the investigation of THC adsorption, and that 10 times more MC Widom insertions $\left(2 \times 10^{7}\right.$ insertions $)$ than MC NVT steps $\left(2 \times 10^{6}\right.$ steps $)$ are needed $\left(2 \times 10^{6}\right.$ steps $)$ to achieve a comparable convergence. We report the full analysis on Mg-MOF-74 in the Supporting Information.

To check for selectivity of THC adsorption in the presence of water vapor, we computed the Henry coefficient of water using MC Widom insertions following the protocol of Moghadam et al. ${ }^{45}$ Point charges for these materials were derived using the extended charge equilibration (EQeq) method, ${ }^{46}$ which we recently benchmarked. ${ }^{47}$ To model water, we used the TIP4P/2005 model $^{48}$ with a unitedatom Lennard-Jones interaction site. We computed the Henry coefficient $\left(k_{\mathrm{H}}\right)$ for water in these materials using $2 \times 10^{6}$ Widom insertions and blocking spheres computed for a probe radius of $1.5 \AA$. As we are including MOFs which have a coordinatively unsaturated metal site (also referred to as open metal site or OMS), one has to consider that modeling interactions using only Coulombic and dispersion interactions often leads to an underestimation of the binding of polar gas molecules at these sites. Our group has spent considerable effort to address this issue by proposing tailor-made corrections to tune the force field for some specific gas-OMS interactions. ${ }^{42,49,50}$ However, a reliable and systematic correction for all combinations of polar molecules (such as water) and OMSs has not yet been proposed due to the complexity of the problem, and therefore, it is of common practice to take the results from standard interactions (e.g., using point charges and off-the-shelf Lennard-Jones parameters) with a grain of salt and analyze overall trends over a large set of structures. $4,51,52$

2.1. Geometric Pore Analysis. Zeo $++^{31}$ was used to compute the most common geometric pore descriptors, including geometric void fraction, accessible surface area, pore limiting diameter (PLD), largest included sphere (LIS), and blocking spheres.

Zeo++ assumes idealized spherical probe molecules for its analysis. This is useful for adsorbates which are approximately spherical or at least have a circular cross-section; however, THC is asymmetric from all view points and a spherical probe is not sufficiently representative of its structure. Here, we introduce another method for determining framework accessibility, applicable to THC (see Figure 1) and similarly asymmetric adsorbates.

Our algorithm utilizes 2-D projections of both the THC molecule and the limiting-diameter pore to calculate the overlap in their crosssectional areas. The cross section of the probe molecule was represented as an ellipse of $7 \times 10 \AA$, which we determined to best match the 2-D projection of THC down its longest axis (Figure 2), using the $\sigma$ Lennard-Jones parameter from $\mathrm{UFF}^{37}$ as the atomic radii.

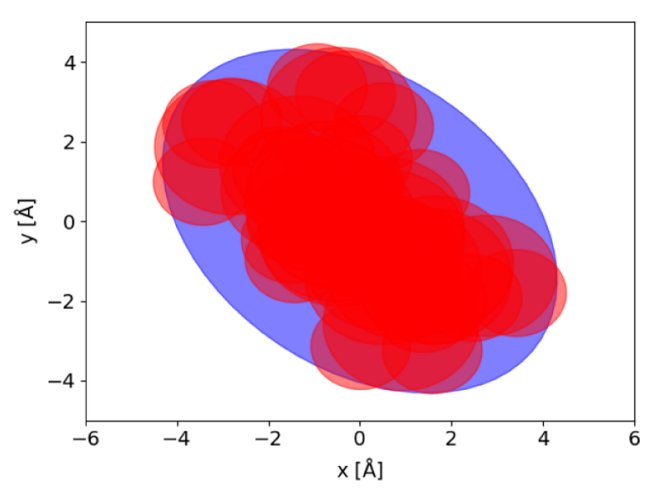

Figure 2. Projection of THC molecule (red circles, radii represent Lennard-Jones $\sigma$ ) overlaid with an ellipse (blue) with minor and major axes of 7 and $10 \AA$, respectively.

In order to compute an equivalent 2-D projection of the pore geometry, we modified a version of the Zeo++ $\operatorname{code}^{53}$ to print coordinates of the Voronoi edge associated with the PLD, $\vec{E}_{\mathrm{PLD}}$. Radii for the projection of framework atoms were also set to the corresponding Lennard-Jones $\sigma$ from UFF. We define at the midpoint of $\vec{E}_{\mathrm{PLD}}$ a plane perpendicular to $\vec{E}_{\mathrm{PLD}}$, denoted $P_{\perp}$. The edges of the Voronoi network define the paths along which an adsorbate can travel in a material, and we thus consider $P_{\perp}$ to be the cross-sectional plane, where the adsorbate encounters the material's PLD. We next project onto this plane all atoms of the framework that the probe would "feel" if it were to pass through. As the rigid head of THC is approximately $10 \AA$ in diameter (Figure 1b), we include in the projection all framework atoms within $5 \AA$ distance from $P_{\perp}$ and within $8 \AA$ radially from the line defined by $\vec{E}_{\perp}$. All atoms within this cylindrical cutoff are projected onto $P_{\perp}$. We evaluate the fit of the elliptical probe in the pore projection, by varying the angle and position of the probe in the middle of the pore and minimizing the area of overlap. ${ }^{54}$

\section{RESULTS}

\subsection{Screening MOF Databases for THC Adsorption.}

We assembled a database of MOFs to screen for THC selectivity, considering the frameworks from the CoRE MOF database, $^{29}$ as well as the MOF-74 analogs from Witman et al. ${ }^{30}$ A large fraction of these porous frameworks can be excluded from our screening on the basis of accessibility: THC, because of its size, can diffuse only through pores that are connected by wide channels. Therefore, we excluded all MOFs with a computed PLD less than $7 \AA$, leaving about $14 \%$ of the frameworks in the original databases (Figure 3). We also removed the CoRE MOFs that have a net charge and contain ions in the pores to counterbalance their charge. The modeling of these counterions requires more advanced techniques as they can move inside the pore. Finally, we excluded six other materials that do not contain any carbon atoms in their structures and therefore cannot be considered MOFs (MARJAQ LETPOR, LETPUX, LETQAE, LETQEI, LETRAF). These probably come from erroneous parsing during the creation of the CoRE MOF database. 

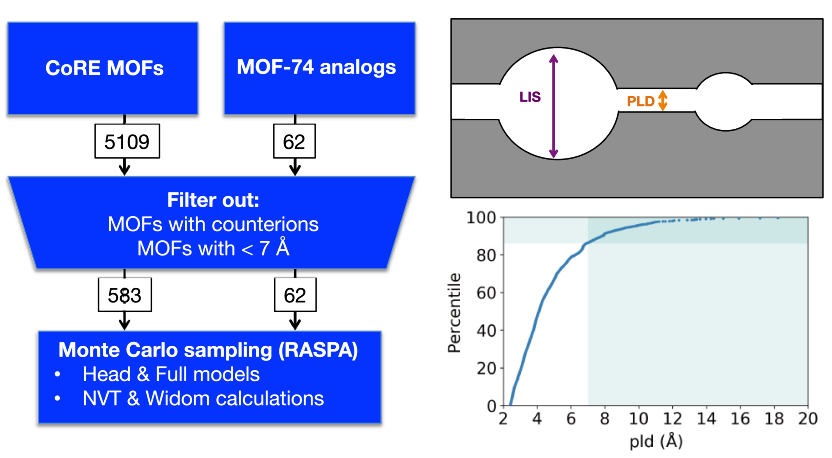

Figure 3. Diagram of screening workflow, including a filter to select only materials with PLD larger than $7 \AA$ and without counterions in the pores.

We chose a lower PLD limit of $7 \AA$ as it corresponds to the narrowest part of the terpenoid group (Figure 1). However, it is still possible that materials with a PLD of $7 \AA$ would not be permeable by a THC molecule, if the perpendicular axis is smaller than the longer axis of THC (with size of roughly 10 A). This motivates our development of the novel "ellipsoid overlap" method, the results of which are discussed in the following section, to determine more accurately the accessibility to THC.

For the remainder of this work, we consider only the subset of 645 MOFs with PLD > $7 \AA$ and without counterions. The distribution of properties of these materials are shown as histograms in Figure S1. All MOF-74 analogs have a PLD between 14 and $45 \AA$, as seen in the inset of the figure. The larger pore size MOFs will allow us to observe the influence of the ligand type for adsorption in MOFs with the same topology.

We start by comparing the Henry coefficient for all MOFs as obtained from Widom insertions using the THC-head and the THC-full models. This will allow us to assess the contribution of the alkyl tail for the adsorption properties in a diverse set of pore topologies. As one can see from Figure 4 the THC-head model, despite its simplicity, leads to a computed Henry coefficient that correlates very well with the full model.

It is interesting to observe the two extremes of low and high Henry coefficient. In frameworks where the Henry coefficient is high (i.e., in more favorable adsorption sites), the inclusion

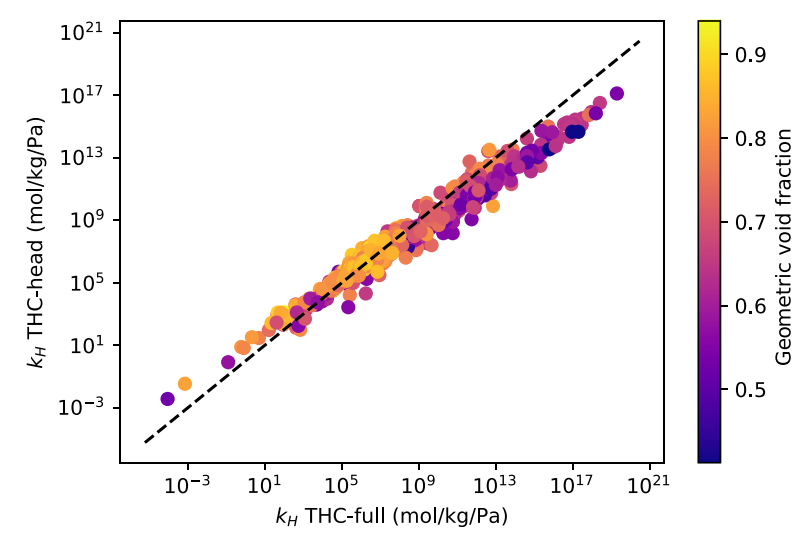

Figure 4. Parity plot of the Henry coefficient for the THC-head and THC-full models. The geometric void fraction of the frameworks is indicated by the coloring. These results are computed from $2 \times 10^{7}$ Widom insertions. of the flexible alkyl tail increases the Henry coefficient from the THC-head model with only a few exceptions. This phenomenon is the foreseeable consequence of adding more atoms, that is, more dispersion interactions with the adsorbent. On the other end, in frameworks where the Henry coefficient is very low, adding the tail makes the Henry coefficient slightly lower. This suggests that these materials with low Henry coefficient have pores where the THC-head already barely fits, and the inclusion of the tail makes these crowded sites even more sterically hindered. Despite these systematic differences, the inclusion of the flexible tail in the model does not significantly change the overall ranking in performance of these MOFs, suggesting that the THC-head model could be a suitably accurate proxy for screening for adsorption properties.

Using both models, we can now evaluate the contribution of the entropy and enthalpy on the Henry coefficient. As the enthalpy of adsorption can be computed more efficiently from an NVT simulation, we are interested to assess if this can be used as a reasonable ranking parameter for THC adsorption. Indeed, we show in the Supporting Information that NVT simulations of THC in MOF-74 already converged after $2 \times$ $10^{6} \mathrm{MC}$ steps, whereas, when probing with Widom insertions, a comparable convergence can be achieved with $2 \times 10^{7}$ insertions. This makes, in practice, the Widom technique roughly 10 times more expensive. Moreover, by comparing the same quantity, the enthalpy of adsorption, $\left(\Delta H_{\mathrm{ads}}=\Delta E_{\mathrm{ads}}-\right.$ $R T$ ), as computed separately by both Widom and NVT, we have a further check on the convergence of the calculations. The entropic and enthalpic contributions to the Henry coefficient are shown in Figure 5, together with the comparison with the enthalpy as computed from NVT simulation.

We can observe from Figure 5 that the main contribution to the Henry coefficient, as expressed in the equation

$$
k_{\mathrm{H}} \cdot \rho_{\mathrm{MOF}}=\frac{1}{R T} \exp \left(\frac{\Delta S_{\mathrm{ads}} \cdot T-\Delta H_{\mathrm{ads}}}{R T}\right)
$$

comes from the enthalpic term. Indeed, considering the entire set of frameworks, the observed range of enthalpies is more than 5 times larger than the entropic range (in Figure 5, we multiplied the entropy by the temperature to have a quantity with the same units of energy as the enthalpy). This gets exponentially enhanced in eq 2 , letting us conclude that the main contribution to the Henry coefficient, for THC adsorption, comes from the adsorption enthalpy. We therefore expect that this quantity provides not only a quick but also an accurate ranking to identify materials with high Henry coefficient for THC.

Considering together the results of Figures 4 and 5, we can conclude that a ranking of materials that is favorable for THC adsorption can be achieved in two ways: (i) by directly comparing the computed Henry coefficient from the most expensive and accurate calculation, that is, the Widom adsorption using the THC-full model, and (ii) by comparing the heat of adsorption from NVT for the THC-head model, a less computationally expensive method. Considering the $10 \times$ speedup due to faster convergence of NVT simulations, and the $1.5 \times$ to $3 \times$ speedup of using the THC-head model (see benchmark calculations in the Supporting Information), the second protocol results up to 30 times faster than the bruteforce calculation of the Henry coefficient with the more realistic and flexible THC-full model. Moreover, Figure 6 
(a)

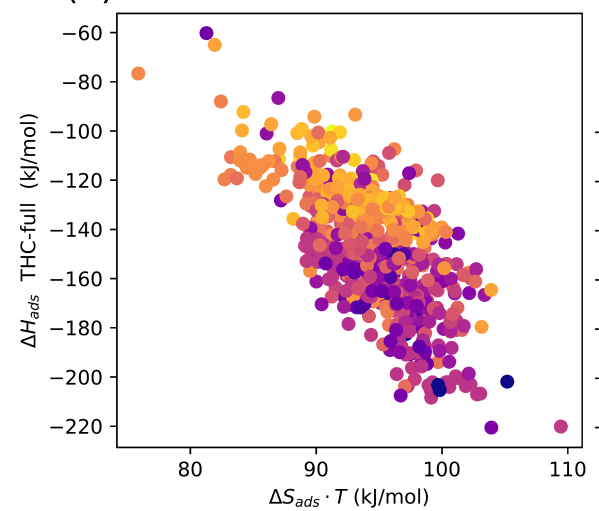

(c)

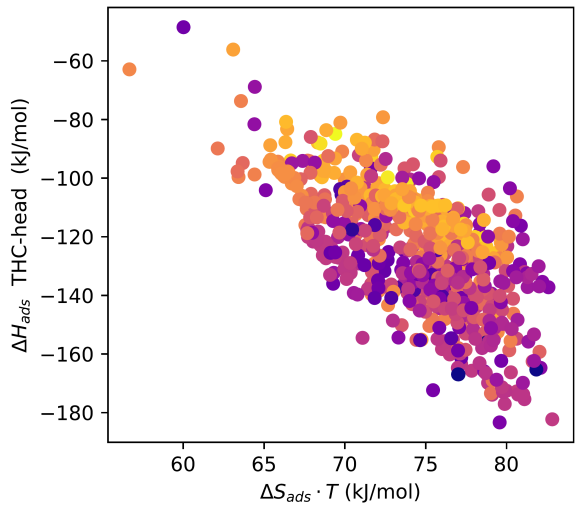

(b)

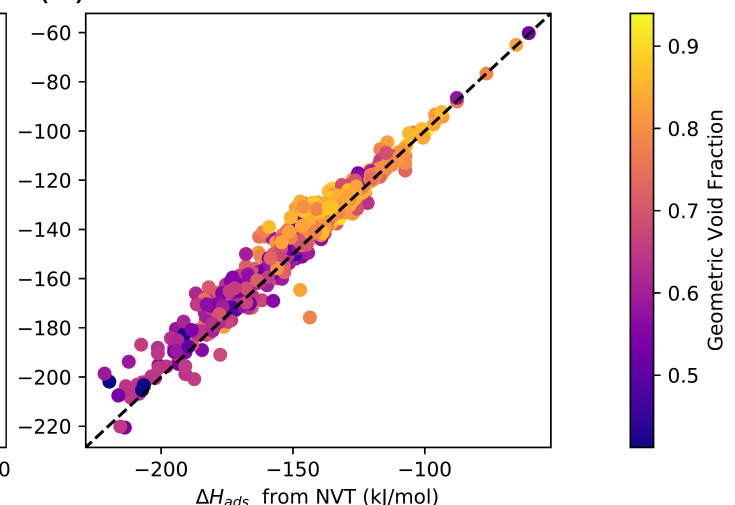

(d)

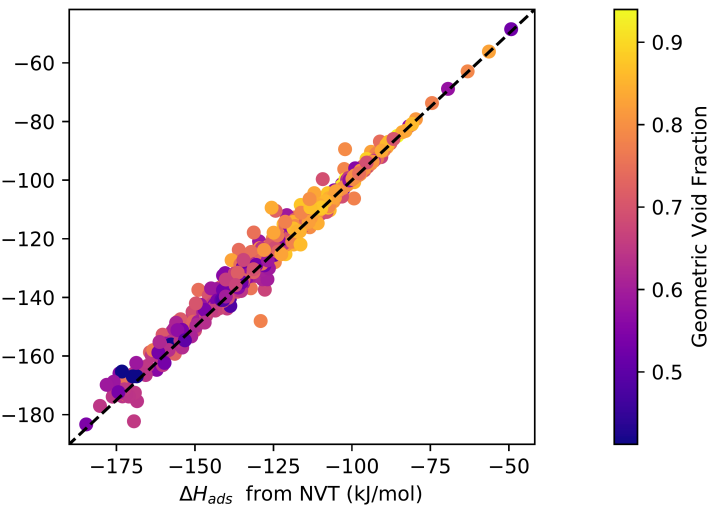

Figure 5. The Henry coefficient is decomposed into its enthalpy and entropy components for the THC-full (a,b) and THC-head (c,d) models. In the parity plots on the right $(\mathrm{b}, \mathrm{d})$, the adsorption enthalpy computed from Widom insertions $(y$-axis) is compared with the same quantity obtained from NVT simulations ( $x$-axis).

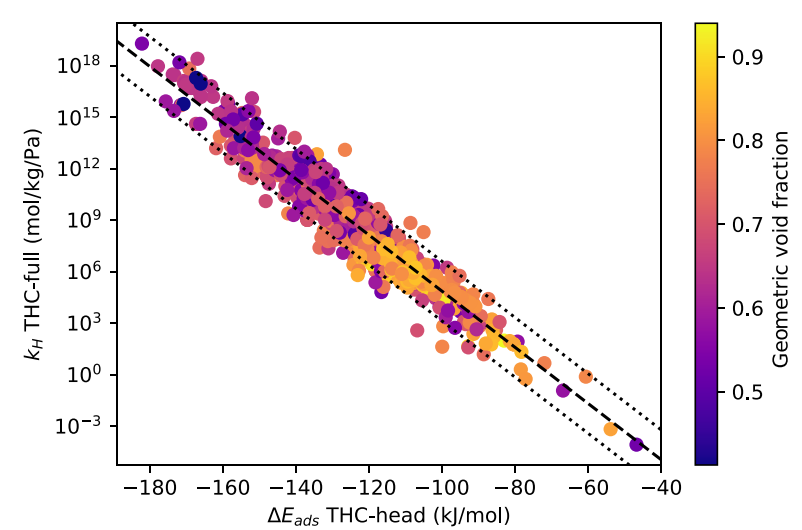

Figure 6. Comparison of the Henry coefficients from Widom insertions using the THC-model and the energy of adsorption from NVT MC simulations for the THC-head model. The empirical relation between the two is drawn with a dashed line, whereas the confidence threshold is shown in dotted lines. This relation has been obtained from linear interpolation.

shows that there is a very strong correlation, and one can relate the two calculations with the empirical relation

$$
k_{\mathrm{H}}^{\text {THC-full }}=\exp \left(-26.41-0.38 \cdot \Delta E_{\mathrm{ads}} \pm 3.0\right)
$$

with $k_{\mathrm{H}}^{\mathrm{THC}-\text { full }}$ in $\mathrm{mol} \mathrm{kg}^{-1} \mathrm{~Pa}^{-1}$ and $\Delta E_{\mathrm{ads}}$ in $\mathrm{kJ} \mathrm{mol}^{-1}$.

Next, we investigate the correlation between adsorption and conventional descriptors, to highlight trends that can be exploited for the rational design of new materials. In Figure S3, we plot the Henry coefficient and the adsorption energy against geometric void fraction, volumetric surface area, PLD, and LIS (as defined in Figure 3).

For most of the descriptors, there is not a clear correlation with Henry coefficient or adsorption energy. The primary interesting feature we can highlight is the similar adsorption energy, around $130 \mathrm{~kJ} \mathrm{~mol}^{-1}$, for all MOFs having large pores and channels (i.e., large PLD and LIS), with many MOF-74 analogs falling in this category. To understand this plateau of adsorption properties, we consider that in large channels and cavities, the pore walls have very large radii of curvature and appear close to flat to an adsorbate. When the THC molecule adsorbs to such a low-curvature surface, the adsorption energy is only influenced by the chemistry of the adjacent surface and not by the shape of the pore. Considering the similar adsorption energy for these structures, we can conclude that the variations in chemistry, for example, between different ligands in MOF-74 analogs, are not significantly affecting the strength of adsorption. Frameworks with smaller PLD have a large range of adsorption energies, extending both lower and higher than the range of adsorption energies for materials with larger PLD. Smaller PLD is correlated with a higher likelihood of finding strongly favorable and unfavorable adsorption energies. The existence of these dual extremes suggests that when pores are small, there is a greater likelihood that the THC molecule will either bind in a very tight, very favorable pocket, or that steric hindrance of an ill-fitting pore will penalize binding. This is consistent with previous work showing similar widening ranges of adsorption energies in small pores for linear and branched alkanes. ${ }^{55}$ 


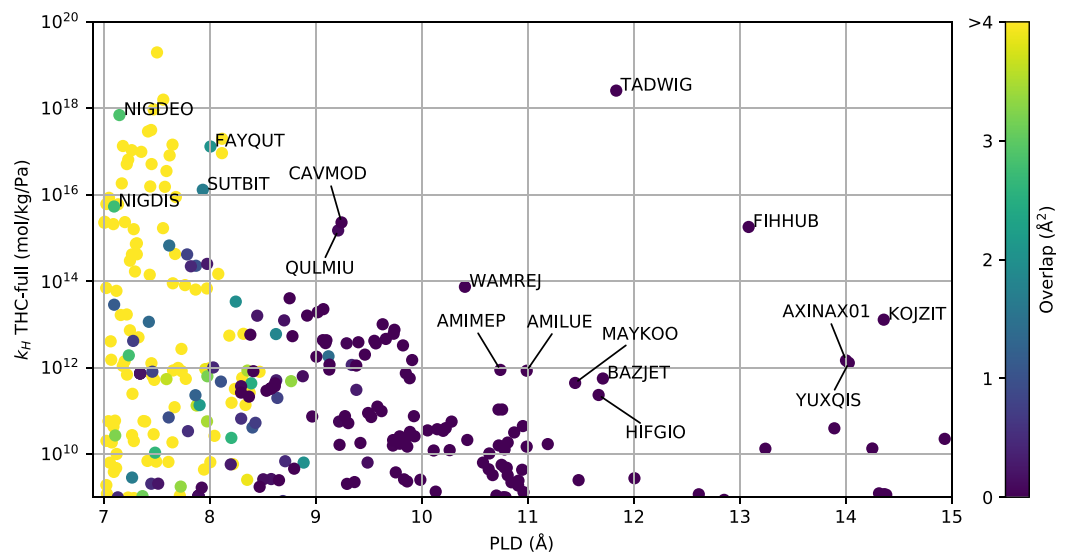

Figure 7. Henry coefficient vs PLD, with point color indicating overlap area. Overlap areas are saturated at $4 \AA^{2}$. Materials mentioned in the text are labeled for reference. We note that the overlap area was only computed explicitly for those materials with PLD from 7 to $10 \AA$; for materials with PLD > $10 \AA$ the overlap area was assumed to be 0 .

3.2. Pore Accessibility to Asymmetric Probe Molecules. We are interested in restricting our screening only to those materials through which THC can percolate, as well as determining whether THC can pass through a particular channel in a material. Most geometric methods to evaluate pore accessibility typically use idealized spherical probe molecules for their analyses. For example, the PLD indicates the size of the largest sphere which can percolate through the material, giving an idea of the barriers to diffusion. This is useful for adsorbates which are approximately spherical or at least have a circular cross-section; however, THC is asymmetric from all view points and is therefore characterized by multiple characteristic lengths; as seen in Figure 1, when the THC molecule is viewed down its longest axis, it has a cross section with dimensions of roughly 10 and $7 \AA$ along its longer and shorter axes, respectively. Therefore, THC should be able to traverse channels with a PLD greater than its longest dimension, whereas it would not be able to fit through a channel whose PLD is smaller than its shortest dimension. For intermediate pore sizes, accessibility depends on the precise pore geometry as compared with the geometry of the probe molecule.

We developed a method which can be used to determine whether an asymmetric molecule can percolate through a porous material, by comparing the shape of the probe molecule to the cross-sectional geometry at the narrowest part of the pore. The algorithm, which is described in detail in the Computational Methods section, computes the minimum area of overlap between the probe and the pore. As atoms are projected as circles with radii equal to their respective Lennard-Jones $\sigma$ parameters, a nonzero overlap area corresponds to interactions in the repulsive regime of the Lennard-Jones potential and thus serves as a proxy for the barrier for diffusion through the pore. We present our results applying this algorithm to our screening database in this section.

The cumulative probability distribution of the overlap area for 412 materials with PLD between 7 and $10 \AA$ is shown in Figure S5. It is not obvious where to draw the line between an accessible and nonaccessible material; however, we determine that $4 \AA$ is a sensible threshold for more accessible pores as the cumulative distribution shows a steep increase just above $4 \AA$. Further examining the probability distribution, we note that $45 \%$ of the materials with PLD between 7 and $10 \AA$ have an overlap of less than $1 \AA^{2}$, and $60 \%$ of materials have an overlap below $4 \AA^{2}$. This allows us to exclude more materials from our consideration of most promising adsorbents, as we show in Figure 7, which plots the Henry coefficient versus PLD of structures with points colored to indicate overlap area, where the color scale extends to $4 \AA$.

This analysis also justifies our choice of a conservative initial cutoff of $7 \AA$ PLD because a higher cutoff might have falsely identified materials such as NIGDEO (PLD = $7.15 \AA$ ) or SUTBIT (PLD $=7.94 \AA$ ) as inaccessible, whereas we find that the overlap areas for these materials is are 2.88 and $1.68 \AA$, respectively, which is below our chosen threshold for accessibility. A visualization of the $2-\mathrm{D}$ projections of the pore (in red) and elliptical probe representing THC (in blue), along with the atomistic representation of the pore, is shown for these two materials in Figure 8.

More generally, this method provides a way to quantify pore accessibility to nonspherical adsorbates in a fast and inexpensive manner for high-throughput screening studies. Although we use it only for studying accessibility in the pore corresponding to a material's PLD, the algorithm can be flexibly applied to any point inside a material.

3.3. Inspection of Promising MOFs for THC Adsorption. Comparing the structures that have the largest Henry coefficients, as shown in Figure 7, we identify three "adsorption motifs" associated with high affinity toward THC. The first motif is the presence of narrow one-dimensional pores characterized by low PLD, which serve as tightly fitting adsorption sites for THC. These tight pores contribute strong dispersion interactions to the binding energy, but also lead to slow diffusion because the THC molecules would need to move through the channel in a single file. Moreover, the structures with low PLD tend to have a high overlap area, indicating that many of these materials may not even be permeable to THC. We refer to this motif as "narrow channels" and one clear example (which is permeable to THC) is SUTBIT (Figure 9).

The second motif is associated with larger channels, with a PLD in the range of $8-11 \AA$, but low void fractions. This characterizes MOFs that contain many framework atoms in the unit cell. As with the "narrow channels" motif, the MOFs displaying the second adsorption motif also exhibit strong framework-adsorbate dispersion interactions, although this is due to the bulkiness of the framework rather than narrow 


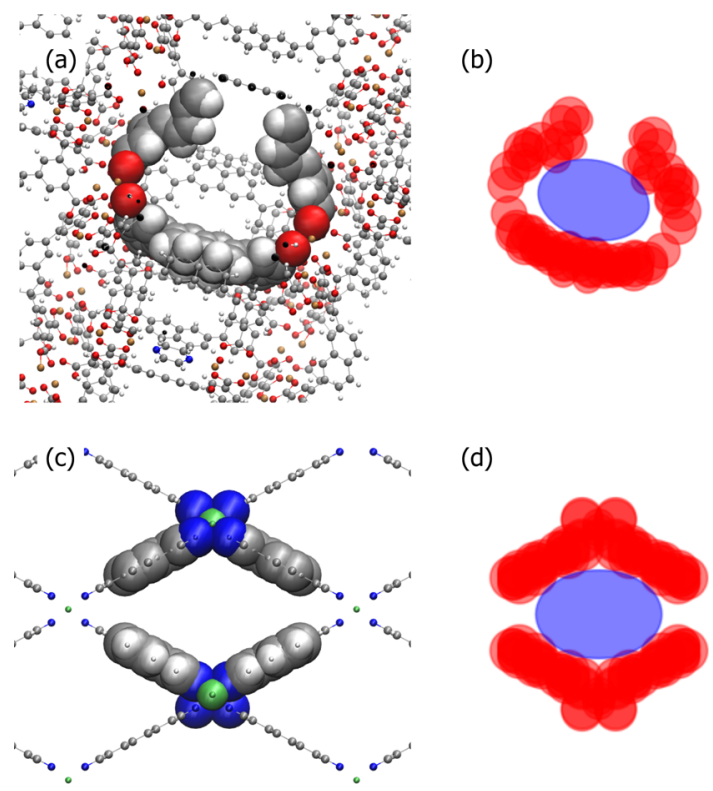

Figure 8. Diagram showing the supercell of each framework with atoms within the cylindrical cutoff and the projection of the pore onto $P_{\perp \vec{E}}$ with the minimally overlapping elliptical probe for NIGDEO $(\mathrm{a}, \mathrm{b})$ and $\operatorname{SUTBIT}(\mathrm{c}, \mathrm{d})$. In the all-atom representations, $\mathrm{C}$ atoms are shown in gray, $\mathrm{H}$ in white, $\mathrm{O}$ in red, $\mathrm{N}$ in blue, $\mathrm{Cu}$ in tan, and $\mathrm{Ni}$ in green. In the projection representations, framework atoms are shown in red, the elliptical probe is shown in blue, and the overlap area (corresponding to the energy barrier of diffusion) is shown in purple.

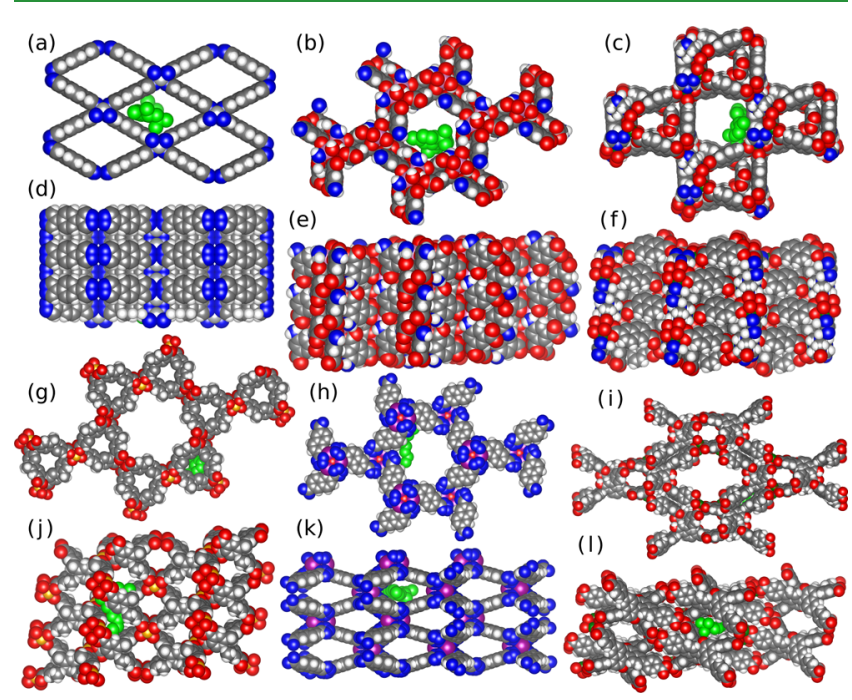

Figure 9. The position of adsorbed THC is showed in the pores of six selected MOFs, as discussed in the main text. MOFs are showed from a top and side view: SUTBIT (a,d), QULMIU (b,e), AMILUE (c,f), $\operatorname{KOJZIT}(\mathrm{g}, \mathrm{j}), \operatorname{BAZJET}(\mathrm{h}, \mathrm{k})$ and TADWIG $(\mathrm{i}, \mathrm{l})$. The full-THC positions have been obtained from equilibrated NVT simulations, and the non-hydrogen elements of the molecule are showed in green. The metals of these MOFs are, respectively: $\mathrm{Ni}$ (silver), In (violet), $\mathrm{Zn}$ (dark green), $\mathrm{Cu}$ (gold), Mn (purple), and $\mathrm{Zn}$ (dark green). The colors of the nonmetal elements are blue for $\mathrm{N}$, grey for $\mathrm{C}$, and red for O.

channels. This motif is found in QULMIU and AMILUE (Figure 9), and we refer to it as "thick walls". Note that there is a smooth transition between narrow channels and thick walls: the first has channels with small PLD, whereas the second has larger channels but also a bulkier framework.
The third and final motif, leading to high THC affinity and lower hindrance of diffusion, represents the most promising feature for THC adsorption: narrow slits, serving as tight binding pockets for THC, which are situated off the sides of wide channels. This configuration is very promising because the THC molecules can diffuse through wider channels (with PLD even above $11 \AA$ ) and adsorb in favorable binding sites that also facilitate their detection by constraining the rotational and translational motion of the molecules. This efficient design resembles a car lot, which has wide lanes to access the narrow spaces where cars are parked: therefore, we gave this motif the name "parking spots". An example of parking spots can be found in KOJZIT and BAZJET (Figure 9).

Once we have defined these three motifs, it is interesting to note that the permeable material with the highest Henry coefficient for THC, TADWIG, exhibits two of them, both parking spots and thick walls (Figure 9). The combination of the two makes it particularly favorable for adsorption and detection of THC. Further examples of these motifs are provided in the Supporting Information.

It is also important to notice that these three adsorption motifs are not restricted to MOF structures: they can in principle be found in other categories of microporous materials such as covalent organic frameworks or zeolites, and they can serve as a guideline to discover or rationally design materials not only for the adsorption of THC, but also for other large molecules with a planar and/or nonspherical shape.

3.4. THC Adsorption in the Presence of Water. As a further computational check of the feasibility of these materials for THC detection from breath, we consider the selectivity of THC adsorption in the presence of water. Exhaled breath is composed of many gaseous species, including water, $\mathrm{CO}_{2}, \mathrm{O}_{2}$, and $\mathrm{N}_{2}$. Of these, selectivity against water is the most important for this application as the polar nature of water and its ability to form hydrogen bonds make it a potential competitor for adsorption sites in MOFs, especially given the humid nature of breath. We should note, however, that other nongaseous species may also play a role in THC sensing from breath. THC in the breath is largely found in aerosol particles formed in small airways, which consist not only of water, but also nonvolatile components such as lipids and proteins. ${ }^{56}$ Thus, a successful breathalyzer technology using MOFs must also take into account how to distinguish THC from this mixture, for example, by using a separation step prior to adsorption. These considerations lie outside the scope of the present study.

In order to determine the affinity of the materials toward water, we computed the Henry coefficient of water using MC Widom insertion simulations. The $\mathrm{THC} / \mathrm{H}_{2} \mathrm{O}$ selectivities (defined as the ratio of $k_{\mathrm{H}}$ of THC to $k_{\mathrm{H}}$ of water) of the materials screened are shown in Figure 10. The materials which have an overlap area of less than $4 \AA$ (i.e., in which THC can percolate through the pores) are colored by the Henry coefficient of THC adsorption, and materials determined to be inaccessible because of a larger overlap are shown in light gray. The most promising materials as identified in the previous section and in the Supporting Information are labeled. We notice that the upper bound of selectivity is higher as the PLD decreases, reflecting the correlation of higher maximum $k_{\mathrm{H} \text {,THC-full }}$ with decreased PLD. In addition, we find that most of the promising materials previously identified also have some of the highest computed $\mathrm{THC} / \mathrm{H}_{2} \mathrm{O}$ selectivities. 


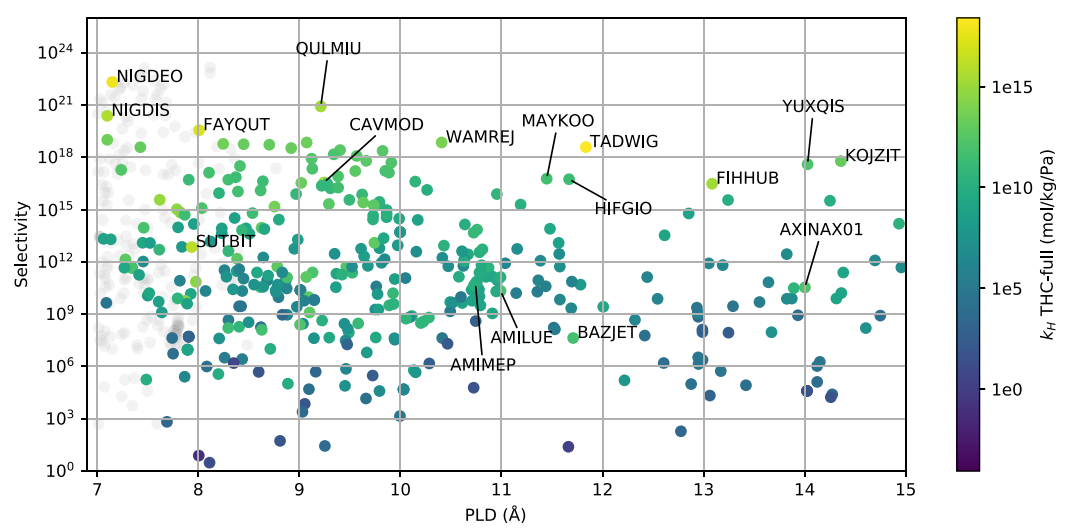

Figure 10. Selectivity of THC adsorption in the presence of water. The colors of the markers indicate the Henry coefficient from Widom insertion.

We next undertake a basic thermodynamic analysis of adsorption in order to determine the $\mathrm{THC} / \mathrm{H}_{2} \mathrm{O}$ selectivity needed in a material for feasible detection of THC from breath. The aerosolized droplets containing THC may in principle equilibrate with water vapor in air. Thus, we assume for our analysis that THC is present in both vapor and aqueous liquid phases, keeping in mind that the surfactant content of the aerosol particles may result in lower vapor concentrations of THC and therefore higher selectivity requirements. Typical concentrations for THC in oral fluid within the first $3 \mathrm{~h}$ of smoking are reported to be on the order of $10 \mu \mathrm{g} \mathrm{L}^{-1}$ to $1 \mathrm{mg}$ $\mathrm{L}^{-1,8}$ nearing the solubility reported for THC in aqueous solutions of $1.05 \mathrm{mg} \mathrm{L}^{-1}$. $^{57}$ The Henry's law constant for THC in water is $0.024318 \mathrm{~Pa} \mathrm{~m}^{3} \mathrm{~mol}^{-1}$. $^{58}$ Assuming that THC at such low liquid concentrations is in the Henry regime, we find that the partial pressure of THC in contact with a THCsaturated aqueous phase is $8.12 \times 10^{-5} \mathrm{~Pa}$ at $25^{\circ} \mathrm{C}$; for oral samples where the liquid-phase concentration varies, the partial pressure could be up to 2 orders of magnitude lower. As breath has a nearly $100 \%$ relative humidity, we can assume that the partial pressure of water is near its saturation pressure, $3.17 \times$ $10^{-3} \mathrm{~Pa}$ at $25^{\circ} \mathrm{C}$, which is $7-9$ orders of magnitude larger than the partial pressure of $\mathrm{THC}$ in breath. If the selectivity of the THC adsorbent is $10^{12}$ or larger, the THC uptake will be at least 1000 times than that of water, even for lower concentrations of THC in breath. According to our model, about $40 \%$ of materials meet this criteria (Figure S10), and several of the promising materials we identified have selectivities many orders of magnitude larger.

This does not mean, however, that materials with a lower selectivity cannot be used in a breath-based THC sensing device. In order to make detection with lower-selectivity materials feasible, the detection device could have a way to remove most of the moisture from the air, perhaps using a very hydrophilic layer first to "dry" the air before it reaches the adsorption and detection module. However, our calculations show that even without a drying step, there are promising candidates with selective adsorption of THC in the presence of water.

\section{CONCLUSIONS}

We have conducted Mone Carlo (MC) simulations to investigate the adsorption of THC in a database of experimentally synthesized and reported structures. In order to increase the efficiency of the screening, we tested a minimal model of THC using only the rigid aromatic head, in addition to a full model of THC with the flexible alkyl tail. We found that using only the THC-head model represents a speedup of 3 times over the THC-full model and is a good proxy for the full model in larger pores. For materials with very small pores, it might be necessary to include the tail in the final evaluation. For a favorable binding site for the THC-head, that is, where the head group fits tightly within the pore, there might not be space for the full molecule, resulting in an unfavorable binding site. Moreover, we show that the adsorption is mainly enthalpy-driven, that is, the binding energy is the main factor that contributes to high affinity. This evidence suggests that the identification of strong binding sites (from a MC NVT simulation) is a good proxy for the Henry coefficient (computable with a more expensive Widom simulation).

We next introduced a method to evaluate the accessibility of pores in the materials by computing the overlap area between the cross-sectional areas of THC and the pore limiting channels in the materials. This method could be useful as an addition to the PLD in determining whether a nonspherical probe can percolate through a material or through a selected pore. By screening for the overlap area in our database, we are able to exclude materials within which THC would have limited accessibility.

Within the THC-accessible materials, we identify three motifs of promising materials for THC adsorption: "narrow channels", "thick walls", and "parking spots". These motifs are not mutually exclusive and, indeed, some materials exhibit more than one. The "parking spot" motif is perhaps the most interesting for an adsorption and sensing application, as the wide channels allow for fast diffusion and the narrow slits bind the adsorbate strongly and limit rotational and translational motions which can lead to noise in optical sensing.

Finally, we computed the Henry coefficient for water to calculate the $\mathrm{THC} / \mathrm{H}_{2} \mathrm{O}$ selectivity of our materials. We conducted a simple thermodynamic analysis to estimate that a selectivity of at least $10^{12}$ would be advantageous for detection of THC directly from humid breath. About $40 \%$ of all THCaccessible materials, and the majority of the most promising materials, fell above this threshold selectivity.

Further investigation is needed to experimentally confirm THC adsorption in the promising materials identified in this study. Moreover, as the goal is to not only adsorb THC but to detect it, experimental and theoretical work can help to identify practicable sensing technologies that would work with these adsorbents.

This study shows that there are materials with potentially selective and favorable adsorption sites for THC. These materials can be used to develop new sensing pathways or 
improve existing ones toward the goal of a breath sensor for THC. More broadly, we present strategies that can be used for the study of adsorption of large molecules in porous materials, including an algorithm to determine pore accessibility to strongly asymmetric molecules such as THC.

\section{ASSOCIATED CONTENT}

\section{S Supporting Information}

The Supporting Information is available free of charge on the ACS Publications website at DOI: 10.1021/acsami.9b13357.

Distribution for the pore descriptors; validation of the THC models on Mg-MOF-74; Henry coefficient and heat of adsorption vs pores descriptors; timing of the different calculations and models; cumulative probability distribution of overlap area; inspection of THC binding sites in additional MOFs; and histogram of THC-water selectivities (PDF)

\section{AUTHOR INFORMATION}

\section{Corresponding Author}

*E-mail: berend.smit@epfl.ch.

\section{ORCID $\odot$}

Daniele Ongari: 0000-0001-6197-2901

Yifei Michelle Liu: 0000-0002-4705-6664

Berend Smit: 0000-0003-4653-8562

\section{Author Contributions}

${ }^{\S}$ D.O. and Y.M.L. contributed equally to this work.

\section{Notes}

The authors declare no competing financial interest.

The input files for RASPA and EQeq programs, and a detailed list of geometrical properties and adsorption results are available on the Materials Cloud Archive (https://archive. materialscloud.org/2019.0046).

\section{ACKNOWLEDGMENTS}

The research is supported by the European Research Council (ERC) under the European Union's Horizon 2020 research and innovation programme (grant agreement no. 666983, MaGic). Y.M.L. acknowledges support from the National Science Foundation Graduate Research Fellowship Program (GRFP). Part of the calculations were enabled by the Swiss National Supercomputing Centre (CSCS), under project ID s761. Part of the research was supported by the NCCR MARVEL, funded by the Swiss National Science Foundation.

\section{REFERENCES}

(1) Hall, W.; Lynskey, M. Evaluating the Public Health Impacts of Legalizing Recreational Cannabis Use in the United States. Addiction 2016, 111, 1764-1773.

(2) Hasin, D. S. US Epidemiology of Cannabis Use and Associated Problems. Neuropsychopharmacology 2018, 43, 195-212.

(3) Rogeberg, O.; Elvik, R. The Effects of Cannabis Intoxication on Motor Vehicle Collision Revisited and Revised. Addiction 2016, 111, $1348-1359$.

(4) Ramaekers, J. G.; Berghaus, G.; van Laar, M.; Drummer, O. H. Dose Related Risk of Motor Vehicle Crashes After Cannabis Use. Drug Alcohol Depend. 2004, 73, 109-119.

(5) Hartman, R. L.; Huestis, M. A. Cannabis Effects on Driving Skills. Clin. Chem. 2013, 59, 478-492.

(6) Couper, F. J.; Logan, B. K. Drugs and Human Performance Fact Sheets, 2014.
(7) Himes, S. K.; Scheidweiler, K. B.; Beck, O.; Gorelick, D. A.; Desrosiers, N. A.; Huestis, M. A. Cannabinoids in Exhaled Breath following Controlled Administration of Smoked Cannabis. Clin. Chem. 2013, 59, 1780-1789.

(8) Marsot, A.; Audebert, C.; Attolini, L.; Lacarelle, B.; Micallef, J.; Blin, O. Comparison of Cannabinoid Concentrations in Plasma, Oral Fluid and Urine in Occasional Cannabis Smokers after Smoking Cannabis Cigarette. J. Pharm. Pharm. Sci. 2016, 19, 411-422.

(9) Kintz, P.; Mura, P.; Jamey, C.; Raul, J.-S. Detection of $\Delta 9$ tetrahydrocannabinol in exhaled breath after cannabis smoking and comparison with oral fluid. Forensic Toxicol. 2017, 35, 173-178.

(10) Kretschmer, C. B.; Wiebe, R. Liquid-Vapor Equilibrium of Ethanol-Toluene Solutions. J. Am. Chem. Soc. 1949, 71, 1793-1797.

(11) Scatchard, G.; Raymond, C. L. Vapor-Liquid Equilibrium. II. Chloroform-Ethanol Mixtures at 35, 45 and 55 ${ }^{\circ}$ J. Am. Chem. Soc. 1938, 60, 1278-1287.

(12) Lovestead, T. M.; Bruno, T. J. Determination of Cannabinoid Vapor Pressures to Aid in Vapor Phase Detection of Intoxication. Forensic Chem. 2017, 5, 79-85.

(13) Beck, O.; Sandqvist, S.; Dubbelboer, I.; Franck, J. Detection of $\Delta$ 9-Tetrahydrocannabinol in Exhaled Breath Collected from Cannabis Users. Journal of Analytical Toxicology 2011, 35, 541-544.

(14) Gordon, M. J.; Jones, L. C.; Lynn, M. S. Devices for Target Substance Detection and Measurement, 2017, https://patentimages. storage.googleapis.com/91/63/24/09f87f7df6b382/US9709581.pdf.

(15) Chong, X.; Kim, K.-J.; Ohodnicki, P. R.; Li, E.; Chang, C.-H.; Wang, A. X. Ultrashort Near-Infrared Fiber-Optic Sensors for Carbon Dioxide Detection. IEEE Sens. J. 2015, 15, 5327-5332.

(16) Chong, X.; Kim, K.-J.; Li, E.; Zhang, Y.; Ohodnicki, P. R.; Chang, C.-H.; Wang, A. X. Near-Infrared Absorption Gas Sensing with Metal-Organic Framework on Optical Fibers. Sens. Actuators, B 2016, 232, 43-51.

(17) Kim, K.-J.; Lu, P.; Culp, J. T.; Ohodnicki, P. R. Metal-Organic Framework Thin Film Coated Optical Fiber Sensors: A Novel Waveguide-Based Chemical Sensing Platform. ACS Sens. 2018, 3, 386-394.

(18) Dronova, M.; Smolianitski, E.; Lev, O. Electrooxidation of New Synthetic Cannabinoids: Voltammetric Determination of Drugs in Seized Street Samples and Artificial Saliva. Anal. Chem. 2016, 88, 4487-4494.

(19) Furukawa, H.; Cordova, K. E.; O’Keeffe, M.; Yaghi, O. M. The Chemistry and Applications of Metal-Organic Frameworks. Science 2013, 341, 1230444.

(20) Zhou, H.-C.; Long, J. R.; Yaghi, O. M. Introduction to MetalOrganic Frameworks. Chem. Rev. 2012, 112, 673-674.

(21) Kitagawa, S.; Kitaura, R.; Noro, S.-i. Functional Porous Coordination Polymers. Angew. Chem., Int. Ed. 2004, 43, 2334-2375.

(22) Eddaoudi, M. Systematic Design of Pore Size and Functionality in Isoreticular MOFs and Their Application in Methane Storage. Science 2002, 295, 469-472.

(23) Moghadam, P. Z.; Li, A.; Wiggin, S. B.; Tao, A.; Maloney, A. G. P.; Wood, P. A.; Ward, S. C.; Fairen-Jimenez, D. Development of a Cambridge Structural Database Subset: A Collection of MetalOrganic Frameworks for Past, Present, and Future. Chem. Mater. 2017, 29, 2618-2625.

(24) Bobbitt, N. S.; Mendonca, M. L.; Howarth, A. J.; Islamoglu, T.; Hupp, J. T.; Farha, O. K.; Snurr, R. Q. Metal-Organic Frameworks for the Removal of Toxic Industrial Chemicals and Chemical Warfare Agents. Chem. Soc. Rev. 2017, 46, 3357-3385.

(25) Matito-Martos, I.; Moghadam, P. Z.; Li, A.; Colombo, V.; Navarro, J. A. R.; Calero, S.; Fairen-Jimenez, D. Discovery of an Optimal Porous Crystalline Material for the Capture of Chemical Warfare Agents. Chem. Mater. 2018, 30, 4571-4579.

(26) Sun, X.; Shao, K.; Wang, T. Detection of Volatile Organic Compounds (VOCs) from Exhaled Breath as Noninvasive Methods for Cancer Diagnosis. Anal. Bioanal. Chem. 2016, 408, 2759-2780.

(27) Qiao, X.; Su, B.; Liu, C.; Song, Q.; Luo, D.; Mo, G.; Wang, T. Selective Surface Enhanced Raman Scattering for Quantitative 
Detection of Lung Cancer Biomarkers in Superparticle@MOF Structure. Adv. Mater. 2018, 30, 1702275.

(28) Gustafson, J. A.; Wilmer, C. E. Intelligent Selection of MetalOrganic Framework Arrays for Methane Sensing via Genetic Algorithms. ACS Sens. 2019, 4, 1586-1593.

(29) Chung, Y. G.; Camp, J.; Haranczyk, M.; Sikora, B. J.; Bury, W.; Krungleviciute, V.; Yildirim, T.; Farha, O. K.; Sholl, D. S.; Snurr, R. Q. Computation-Ready, Experimental Metal-Organic Frameworks: A Tool to Enable High-Throughput Screening of Nanoporous Crystals. Chem. Mater. 2014, 26, 6185-6192.

(30) Witman, M.; Ling, S.; Anderson, S.; Tong, L.; Stylianou, K. C.; Slater, B.; Smit, B.; Haranczyk, M. In silico design and screening of hypothetical MOF-74 analogs and their experimental synthesis. Chem. Sci. 2016, 7, 6263-6272.

(31) Willems, T. F.; Rycroft, C. H.; Kazi, M.; Meza, J. C.; Haranczyk, M. Algorithms and Tools for High-Throughput Geometry-Based Analysis of Crystalline Porous Materials. Microporous Mesoporous Mater. 2012, 149, 134-141.

(32) VandeVondele, J.; Krack, M.; Mohamed, F.; Parrinello, M.; Chassaing, T.; Hutter, J. Quickstep: Fast and Accurate Density Functional Calculations Using a Mixed Gaussian and Plane Waves approach. Comput. Phys. Commun. 2005, 167, 103-128.

(33) Perdew, J. P.; Burke, K.; Ernzerhof, M. Generalized Gradient Approximation Made Simple. Phys. Rev. Lett. 1996, 77, 3865-3868.

(34) Goedecker, S.; Teter, M.; Hutter, J. Separable Dual-space Gaussian Pseudopotentials. Phys. Rev. B: Condens. Matter Mater. Phys. 1996, 54, 1703-1710.

(35) VandeVondele, J.; Hutter, J. Gaussian Basis Sets for Accurate Calculations on Molecular Systems in Gas and Condensed Phases. J. Chem. Phys. 2007, 127, 114105.

(36) Manz, T. A.; Limas, N. G. Introducing DDEC6 Atomic Population Analysis: Part 1. Charge Partitioning Theory and Methodology. RSC Adv. 2016, 6, 47771-47801.

(37) Rappe, A. K.; Casewit, C. J.; Colwell, K. S.; Goddard, W. A.; Skiff, W. M. UFF, a Full Periodic Table Force Field for Molecular Mechanics and Molecular Dynamics Simulations. J. Am. Chem. Soc. 1992, 114, 10024-10035.

(38) Martin, M. G.; Siepmann, J. I. Transferable Potentials for Phase Equilibria. 1. United-Atom Description of n-Alkanes. J. Phys. Chem. B 1998, 102, 2569-2577.

(39) Siepmann, J. I.; Frenkel, D. Configurational Bias Monte Carlo: a New Sampling Scheme for Flexible Chains. Mol. Phys. 1992, 75, 59-70.

(40) Dubbeldam, D.; Calero, S.; Ellis, D. E.; Snurr, R. Q. RASPA: Molecular Simulation Software for Adsorption and Diffusion in Flexible Nanoporous Materials. Mol. Simul. 2016, 42, 81-101.

(41) Rosi, N. L.; Kim, J.; Eddaoudi, M.; Chen, B.; O'Keeffe, M.; Yaghi, O. M. Rod Packings and Metal-Organic Frameworks Constructed from Rod-Shaped Secondary Building Units. J. Am. Chem. Soc. 2005, 127, 1504-1518.

(42) Dzubak, A. L.; Lin, L.-C.; Kim, J.; Swisher, J. A.; Poloni, R.; Maximoff, S. N.; Smit, B.; Gagliardi, L. Ab Initio Carbon Capture in Open-Site Metal-Organic Frameworks. Nat. Chem. 2012, 4, 810-816.

(43) Geier, S. J.; Mason, J. A.; Bloch, E. D.; Queen, W. L.; Hudson, M. R.; Brown, C. M.; Long, J. R. Selective adsorption of ethylene over ethane and propylene over propane in the metal-organic frameworks $\mathrm{M}_{2}$ (dobdc) ( $\left.\mathrm{M}=\mathrm{Mg}, \mathrm{Mn}, \mathrm{Fe}, \mathrm{Co}, \mathrm{Ni}, \mathrm{Zn}\right)$. Chem. Sci. 2013, 4, 2054.

(44) Kapelewski, M. T.; Geier, S. J.; Hudson, M. R.; Stück, D.; Mason, J. A.; Nelson, J. N.; Xiao, D. J.; Hulvey, Z.; Gilmour, E.; FitzGerald, S. A.; Head-Gordon, M.; Brown, C. M.; Long, J. R. $\mathrm{M}_{2}(\mathrm{~m}-$ dobdc) $(\mathrm{M}=\mathrm{Mg}, \mathrm{Mn}, \mathrm{Fe}, \mathrm{Co}, \mathrm{Ni})$ Metal-Organic Frameworks Exhibiting Increased Charge Density and Enhanced $\mathrm{H}_{2}$ Binding at the Open Metal Sites. J. Am. Chem. Soc. 2014, 136, 12119-12129.

(45) Moghadam, P. Z.; Fairen-Jimenez, D.; Snurr, R. Q. Efficient Identification of Hydrophobic MOFs: Application in the Capture of Toxic Industrial Chemicals. J. Mater. Chem. A 2016, 4, 529-536.

(46) Wilmer, C. E.; Kim, K. C.; Snurr, R. Q. An Extended Charge Equilibration Method. J. Phys. Chem. Lett. 2012, 3, 2506-2511.
(47) Ongari, D.; Boyd, P. G.; Kadioglu, O.; Mace, A. K.; Keskin, S.; Smit, B. Evaluating Charge Equilibration Methods To Generate Electrostatic Fields in Nanoporous Materials. J. Chem. Theory Comput. 2019, 15, 382-401.

(48) Abascal, J. L. F.; Vega, C. A General Purpose Model for the Condensed Phases of Water: TIP4P/2005. J. Chem. Phys. 2005, 123, 234505.

(49) Mercado, R.; Vlaisavljevich, B.; Lin, L.-C.; Lee, K.; Lee, Y.; Mason, J. A.; Xiao, D. J.; Gonzalez, M. I.; Kapelewski, M. T.; Neaton, J. B.; Smit, B. Force Field Development from Periodic Density Functional Theory Calculations for Gas Separation Applications Using Metal-Organic Frameworks. J. Phys. Chem. C 2016, 120, 12590-12604.

(50) Ongari, D.; Tiana, D.; Stoneburner, S. J.; Gagliardi, L.; Smit, B. Origin of the Strong Interaction between Polar Molecules and Copper(II) Paddle-Wheels in Metal Organic Frameworks. J. Phys. Chem. C 2017, 121, 15135-15144.

(51) Wilmer, C. E.; Farha, O. K.; Bae, Y.-S.; Hupp, J. T.; Snurr, R. Q. Structure-property relationships of porous materials for carbon dioxide separation and capture. Energy Environ. Sci. 2012, 5, 9849.

(52) Li, W.; Rao, Z.; Chung, Y. G.; Li, S. The Role of Partial Atomic Charge Assignment Methods on the Computational Screening of Metal-Organic Frameworks for $\mathrm{CO}_{2}$ Capture under Humid Conditions. ChemistrySelect 2017, 2, 9458-9465.

(53) Liu, Y. M. michelleliu/zeoplusplus: Modified Extended Output Version, 2019, https://zenodo.org/record/3247355.

(54) Liu, Y. M. michelleliu/geometric-overlap: Development Version, 2019, https://zenodo.org/record/3247347.

(55) Liu, Y. M.; Smit, B. Predicting Product Distribution of Propene Dimerization in Nanoporous Materials. ACS Catal. 2017, 7, 39403948.

(56) Bake, B.; Larsson, P.; Ljungkvist, G.; Ljungström, E.; Olin, A. Exhaled particles and small airways. Respir. Res. 2019, 20, 8.

(57) Garrett, E. R.; Hunt, C. A. Physicochemical Properties, Solubility, and Protein Binding of $\Delta 9$-Tetrahydrocannabinol. J. Pharm. Sci. 1974, 63, 1056-1064.

(58) HSDB. $\Delta$ 9-Tetrahydrocannabinol CASRN: 1972-08-3, 2018, https://toxnet.nlm.nih.gov/cgi-bin/sis/search/r?dbs+hsdb:@term+@ rn+@rel+1972-08-3. 